\title{
Characterization of methylation patterns associated with lifestyle factors and vitamin D supplementation in a healthy elderly cohort from Southwest Sweden
}

Maria Araceli Diaz Cruz

Jönköping University

Benjamin Ulfenborg

University of Skövde

Peter Blomstrand

Jönköping University

Maria Faresjö

Chalmers University of Technology

Fredrik Ståhl

Borås University

Sandra Karlsson ( $\nabla$ sandra.karlsson@ju.se )

Jönköping University

\section{Research Article}

Keywords: healthy elderly, lifestyle, Vitamin D, physical activity, methylation, epigenetics

Posted Date: March 23rd, 2022

DOl: https://doi.org/10.21203/rs.3.rs-1391526/v2

License: (9) (i) This work is licensed under a Creative Commons Attribution 4.0 International License.

Read Full License 


\section{Abstract}

Background: Numerous studies have shown that lifestyle factors, such as regular physical activity and vitamin D intake, may remarkably improve overall health and mental wellbeing. This is especially important in older adults whose vitamin D deficiency occurs with a high prevalence. This study aimed to examine the influence of lifestyle and vitamin D on global DNA methylation patterns in an elderly cohort in Southwest of Sweden. We also sought to examine the methylation levels of specific genes involved in vitamin D's molecular and metabolic activated pathways.

Methods and results: We performed a genome wide methylation analysis, using Illumina Infinium DNA Methylation EPIC 850kBeadChip array, on 277 healthy individuals from Southwest Sweden at the age of 70-95. The study participants also answered queries on lifestyle, vitamin intake, heart medication, and estimated health. Vitamin D intake did not in general affect methylation patterns, which is in concert with other studies. However, when comparing the group of individuals taking vitamin supplements, including vitamin $D$, with those not taking supplements, a difference in methylation in the SCL25A24 gene was found. This confirms a previous finding, where changes in expression of SLC25A24 were associated with vitamin $\mathrm{D}$ treatment in human monocytes. The combination of vitamin $\mathrm{D}$ intake and high physical activity increased methylation of genes linked to regulation of vitamin D receptor pathway, the Wntpathway and general cancer processes.

Conclusions: To our knowledge, this is the first study detecting epigenetic markers associated with the combined effects of vitamin $D$ supplementation and high physical activity. These results deserve to be further investigated in an extended, interventional study cohort, where also the levels of 25(OH)D can be monitored.

\section{Background}

Individual genetic background and environmental factors are interlaced to lifestyle in determining individual health status. The concept of lifestyle is used to describe the "typical way of life or manner of living characteristic of an individual or group" [1]. Increasing evidence demonstrates that environmental and lifestyle factors influence epigenetic mechanisms, such as DNA methylation, which is responsible for activation or repression of the expression of specific genes [2]. Common lifestyle factors known to influence DNA methylation are diet, behaviour, stress, physical activity, psychological stress, smoking, and alcohol consumption [2].

Vitamin $D$ is a group of fat-soluble secosteroids that act as hormones in the human body and can either be ingested in the diet, taken as supplements, or synthesized in the skin under sunlight exposure [3]. Vitamin D has an essential role in human health since its deficiency correlates with various health problems, including depression, cognitive impairment, osteoporosis, cardiovascular disease, hypertension, type 2 diabetes, and cancer [4,5]. Moreover, certain groups have a higher prevalence of vitamin D deficiency, such as high pigmented individuals, obese, hospitalized patients, and especially the 
elderly [6]. In the United States, $35 \%$ of adults and $61 \%$ of the elderly are vitamin D deficient. In Europe, the percentages are lower in adults $(2-30 \%)$ but $80 \%$ in the institutionalized elderly [7]. The elderly are at risk of lower vitamin D levels due to decreased cutaneous synthesis, bioavailability, and vitamin D intake, as well as other lifestyle changes [6,7]. The metabolism of vitamin D is a multistep process involving several receptors and enzymes. Ergocalciferol (vitamin $D_{2}$ ) and cholecalciferol (vitamin $D_{3}$ ) are converted by calciol-25-hydroxylase (CYP2R1) into 25-hydroxycholecalciferol (25(OH)D; calcidiol) [8]. Calcidiol-1ahydroxylase (CYP27B1 gene) then converts 25(OH)D into 1,25(OH)D, the biologically active ligand for the vitamin D receptor (VDR) and the protein disulfide-isomerase A3 (PDIA3) receptors. The majority of circulating $25(\mathrm{OH}) \mathrm{D}$ and $1,25(\mathrm{OH}) \mathrm{D}$ in the blood is bound to the vitamin $\mathrm{D}$ binding protein (DBP) [35]. The vitamin D-DBP complex is latterly degraded, releasing vitamin D metabolites for physiological action or metabolism [35, 36]. A 24-hydroxylase enzyme (CYP24A1 gene) is in charge of the inactivation of both $25(\mathrm{OH}) \mathrm{D}$ and $1,25(\mathrm{OH}) \mathrm{D}$ via hydroxylation [8]. The active vitamin $\mathrm{D}$ metabolite, 1,25(OH)D or calcitriol, is well known for its effect on gene regulation via its action on the VDR [1]. However, the potential for vitamin $\mathrm{D}$ to regulate gene expression through DNA methylation is currently under investigation [2]. Several epigenetic markers have been shown to be influenced by vitamin $D$ supplementation $[9,10]$, whereas other studies did not find any significant associations after statistical adjustment or only weak associations [11-14]. So far, the results of the analysis of methylation of vitamin D receptors and associated metabolic enzymes are ambiguous [14-16].

Physical activity and regular exercise can remarkably improve overall health and mental wellbeing. There is strong evidence that physical activity contributes to increased body function, reduced impairment, independent living, and improved quality of life in the elderly [17]. Regular exercise is also involved in telomere maintenance and the regulation of DNA methylation levels $[18,19]$. Interestingly, even a single bout of exercise (also termed "acute exercise") has been shown to alter global DNA methylation and specific genes' promoter methylation in skeletal muscle [20]. Combining vitamin D and physical activity has demonstrated synergistic effects in the sarcopenic elderly, such as increasing fat-free mass, strength and functionality, and decreased inflammation [21]. An increase in physical activity has been associated with an increase of circulating $25(\mathrm{OH}) \mathrm{D}$ in men, women, adolescents, and the elderly $[22,23]$. The mechanisms behind these effects are still unknown, but muscle cells can take up 25(OH)D from blood and store it by binding with vitamin D binding protein (DBP) during physical activity [24]. Since synergistic effects of vitamin $D$ and physical activity have been demonstrated, this combination may elicit changes in DNA methylation.

Chronological age has been shown to have a profound effect on DNA methylation levels, and several epigenetic markers have been suggested to produce estimations of biological age (referred to as epigenetic age) [25]. However, not much is known about the relationship between epigenetic aging rates and lifestyle factors, such as diet, alcohol consumption and physical activity.

The present study is part of a more extensive cohort study of healthy individuals aged 70-95 in Southwest Sweden, previously described by Gillsjö et al. [26]. The main purpose of this study was to investigate to which extent healthy aging depends on a conscious or unconscious adaption of a lifestyle 
that matches their genetic predisposition to, for example, different pathological conditions or other health issues. Further, this study aimed to investigate how lifestyle factors affect global methylation patterns and, thus, epigenetic age.

Global methylation patterns associated with vitamin D and combined with other lifestyle habits, such as physical activity, have not been extensively studied in healthy elderly populations. These analyses may be meaningful in the elderly, where vitamin D deficiency and sarcopenia are frequent, and with a higher prevalence of diseases linked to suboptimal vitamin $D$ status. The current study examined the influence of lifestyle factors (physical activity, smoking and alcohol) and vitamin D on global DNA methylation in the healthy elderly cohort mentioned above. Vitamin D intake was analysed through three different sources: Dietary, supplementation and synthesis. We investigated dietary intake through fish intake due to its high content in vitamin D. Synthesis of vitamin D was evaluated from sunlight exposure frequency. The use of sunscreen was also investigated since sun protection may also affect vitamin $D$ synthesis negatively. Furthermore, we analysed methylation levels of specific genes involved in vitamin D's molecular and metabolic activated pathways.

\section{Results}

\section{Differentially methylated probes (DMPs) associated with confounding variables: Gender, age, smoking and alcohol habits}

PCA analysis was performed on the pre-processed methylation dataset to identify potential batch effects in the study (Fig. 1).

The PCA plot shown in Fig. 1A revealed two distinct clusters of samples that corresponded to the gender of participants. 4,329 differentially methylated probes (DMPs) and 591 differentially methylated regions (DMRs) were identified, separating females from males (Fig. 1B).

Age (Table 3) as well as alcohol habits (SD/week and frequency, Table S1A, Additional file 2) did not show any association with changes in methylation. When analysing smoking habits, 11 DMPs were identified when comparing the group defined as "High" $(n=7)$ with "None" $(n=149$, Table S3, Additional file 3).

\section{DMPs associated with Vitamin D supplements, fish intake and sun exposure}

Participants within the cohort answered whether they were taking vitamin D supplementation or not (Yes/No) and to specify if the vitamin D was part of a multivitamin complex (MC) (Table S1A, Additional file 2). Only one DMP and one DMR were found when comparing the group taking vitamin $D$ as part of a $M C(n=8)$ with exclusively vitamin $D(n=33)$, or with not intake of vitamin $D$, respectively $(n=229, F D R=$ 
$0.03, \beta=0.2$, Table S4, Additional file 3). The DMP was located in the promoter region of the SLC25A24 gene in the region from the transcription start site (TSS) to -200 nucleotides upstream (TSS200). No DMPs were found when analysing dietary vitamin $D$ from fish exclusively (Table S1B, Additional file 2). Vitamin D synthesis through sunlight exposure or inhibition by the influence of sunscreen (Table S1B, Additional file 2) could not be associated with any methylation changes. The combination of vitamin $D$ supplementation with sun exposure and/or dietary vitamin D did not result in any significant changes in methylation either.

\section{DMPs associated with physical activity}

Participants within the cohort also answered questions regarding physical activity during winter and summer, respectively. Questions were ranked from 1 to $6(1=$ almost no physical and $6=$ hard regularly training several times a week). The physical activity was considered "High" when the sum of the ranks for the summer and winter exercise was $>=9$ and $<=12(n=31)$; "Intermediate," when it was = $8(n=104)$; and "Low"; when it was $<8(n=134)$. No significant differences in methylation were found from pairwise comparisons between the groups.

Two DMPs in intergenic regions were found when comparing individuals reporting moderate to high physical activity $(n=262)$ with very low or to almost no physical activity $(n=7$, Table S5, Additional file 3).

\section{DMPs associated with Vitamin D supplements and high physical activity}

A total of 357 DMPs, corresponding to 221 genes and 108 intergenic regions, and 9 DMRs, were identified when comparing the group taking vitamin $\mathrm{D}$ supplements and exerting high level of physical activity $(\mathrm{n}=$ 7) with the group of individuals not taking vitamin $D$ supplements and exerting low levels of physical activity ( $n=117$, Table S6-S7, Additional file 3 ). Table 1 shows the 20 DMPs with the highest statistical significance. Totally 74 out of the 357 DMPs $(21 \%)$ were located in promoter regions (Table S8, Additional file 3). No differences in methylation were found between the group taking vitamin $D$ supplementation and intermediate physical activity $(n=17)$ compared with not taking vitamin $D$ supplements and exerting only low level of physical activity $(n=117)$. 
Table 1

DMPs in the group of individuals taking vitamin D supplements and exerting high level of physical activity.

\begin{tabular}{|c|c|c|c|c|}
\hline Gene & Probe & Chr & $F D R$ & $\beta$ \\
\hline$D A B 2$ & cg21886364 & 5 & $6.5 \mathrm{E}-07$ & 0.28 \\
\hline COL23A1 & cg07145979 & 5 & 7E-07 & 0.28 \\
\hline SOD3 & cg17573292 & 4 & 2.7E-06 & 0.34 \\
\hline$S K I$ & cg13488570 & 1 & 4.5E-06 & 0.23 \\
\hline LPIN1 & cg00523161 & 2 & 4.5E-06 & 0.34 \\
\hline Intergenic region & cg24792289 & 5 & 4.5E-06 & 0.27 \\
\hline Intergenic region & cg23244910 & 6 & 6.9E-06 & 0.28 \\
\hline GLI3 & cg06310816 & 7 & 2.1E-05 & 0.20 \\
\hline LOC100132111, C2CD4D & cg26426745 & 1 & 2.4E-05 & 0.35 \\
\hline$R P H 3 A L$ & cg15295273 & 17 & 2.4E-05 & 0.31 \\
\hline RTN4RL 1 & cg08454053 & 17 & $3.2 \mathrm{E}-05$ & 0.24 \\
\hline KIAA0319L & cg22698544 & 1 & 7.1E-05 & 0.26 \\
\hline Intergenic region & cg05964935 & $Y$ & 7.1E-05 & -0.29 \\
\hline$N K X 2-8$ & cg20008148 & 14 & 8.8E-05 & 0.36 \\
\hline NTM & cg04934246 & 11 & 2.3E-04 & 0.21 \\
\hline Intergenic region & cg16668359 & 2 & 2.3E-04 & 0.31 \\
\hline NFIX & cg20116828 & 19 & $3 E-04$ & 0.38 \\
\hline Intergenic region & cg20586840 & 4 & 3.3E-04 & -0.21 \\
\hline IQSEC1 & cg16562217 & 3 & 3.7E-04 & 0.56 \\
\hline Intergenic region & $\operatorname{cg} 13100965$ & 7 & $3.8 \mathrm{E}-04$ & 0.31 \\
\hline
\end{tabular}

* FDR: False discovery rate, Chr: chromosome, $\beta$ : beta value difference

\section{Methylated genes induced by vitamin D and high physical activity are associated with global regulation of transcription}

Functional annotation analysis was performed with PANTHER for all 221 genes containing DMPs for the vitamin $D$ and high physical activity group. Table 2 shows the most enriched terms $(F D R<0.05)$ for 
biological process (BP) and molecular function (MF).

Table 2

Functional annotation analysis of differentially methylated genes associated with vitamin $D$ supplements and high physical activity group.

\begin{tabular}{|lllc|}
\hline GO category & Term & $\begin{array}{l}\text { \# } \\
\text { genes }\end{array}$ & FDR \\
\hline $\begin{array}{l}\text { Molecular function } \\
\text { (MF) }\end{array}$ & $\begin{array}{l}\text { RNA polymerase II cis-regulatory region sequence-specific } \\
\text { DNA binding }\end{array}$ & 30 & 0.02 \\
\cline { 2 - 4 } & $\begin{array}{l}\text { DNA-binding transcription activator activity, RNA } \\
\text { polymerase Il-specific }\end{array}$ & 17 & 0.03 \\
\cline { 2 - 4 } & Protein binding & 181 & 0.04 \\
\hline $\begin{array}{l}\text { Biological process } \\
\text { (BP) }\end{array}$ & $\begin{array}{l}\text { Positive regulation of transcription by RNA polymerase II } \\
\text { promoter }\end{array}$ & 35 & 0.002 \\
\cline { 2 - 4 } & Cell adhesion & 27 & 0.007 \\
\cline { 2 - 4 } & Regulation of cell population proliferation & 36 & 0.03 \\
\hline
\end{tabular}

* \# genes: Number of genes, FDR: False discovery rate

The enriched terms reported by PANTHER (BP and MF) were related to processes and mechanisms related to transcriptional regulation (Table 2). The most significant MF and BP were related to a regulation of the transcription in the cis-regulatory element or promoter of the RNA polymerase II. There were 22 genes overlapping between MF and BP: HHEX, DLX1, NFIX, ZNF721, PLAGL1, TFAP2E, TBX1, SKI, HOXD13, FOSB, RUNX1, DLX5, FOXI1, GLI3, ZNF639, TFCP2, OTX1, NKX2-8, RFX5, NR2F1, NFATC4, and SPI1.

Network analysis with GeneMania showed that genes related to hypomethylated probes and their associated genes were involved in "Sodium ion transport" processes (Figure S1, Additional file 4, FDR = 0.005-0.03).

\section{Methylated genes induced by Vitamin D and high physical activity are linked to the regulation of the Vitamin D receptor, Wnt signaling, and cancer pathways}

Genes corresponding with differentially hypermethylated and hypomethylated probes, for the vitamin D and high physical activity group were separately input into GeneCodis to analyse the main regulated pathways. Figure 2 shows the main regulated pathways for the genes corresponding with differentially hypermethylated positions. The three most significant pathways obtained from WikiPathways analyses were: Vitamin D receptor, transforming growth factor beta (TGF- $\beta$ ), and the Wnt signaling pathway 
(Fig. 2A). The three most significant cellular processes/pathways extracted from KEGG were cancer, stem cell pluripotency, and neurodegeneration in multiple diseases (Fig. 2B).

\section{Methylation status of vitamin D-related genes}

Methylation levels of the 80 vitamin D-related genes, including vitamin D receptors and metabolic enzymes among others (Table S2, Additional file 2) showed weak correlations with different factors analysed in the study. The methylation levels of $12 \mathrm{CpG}$ sites in 8 of the 80 vitamin D-related genes, showed a correlation value $\mathrm{r} 2 \approx 0.2$, (Table S9, Additional file 4). Age showed a negative association with the methylation levels of $R X R A(\mathrm{r} 2=-0.22$, adjusted $P$ value $=0.001), P P A R G C 1 B(\mathrm{r} 2=-0.27$, adjusted $P$ value $=0.000009)$, SMARCA4 (r2= -0.21, adjusted $P$ value $=0.00080)$ and SMARCC1 $(\mathrm{r} 2=-0.21$, adjusted $P$ value $=0.00080)($ Fig. 3). On the contrary, age showed a positive association with methylation levels of COPS2 (r2 $=-0.20$, adjusted $P$ value $=0,00093$ ) and ACSL 1 ( $r 2=-0.20$, adjusted $P$ value $=0.00083)($ Table s9, Additional file 4 \& Fig. 3).

Methylation status of the 80 vitamin D-related genes did not change with vitamin D supplementation (FDR = 0.5-0.9). There were no significant changes in methylation associated with the rest of the binary factors, including vitamin supplements, medicines (lipids, heart, and blood pressure), self-reported health status (SRHS) and alcohol (frequency/week).

\section{Discussion}

Global methylation patterns associated with intake of vitamin D supplements combined with other lifestyle habits, such as physical activity, have not been thoroughly studied in elderly populations. Herein, we assessed the influence of lifestyle habits, including vitamin D intake, on global DNA methylation patterns in an elderly cohort in Southwest of Sweden. Further, we examined the methylation status of vitamin D-specific genes involved in vitamin D's molecular and metabolic activated pathways.

In the present study, vitamin D intake from diet and supplementation and sunlight exposure or the use of sunscreen did not affect, in general, the methylation patterns in the elderly. Effects in methylation could only be observed when comparing groups with individuals taking vitamin supplements, including vitamin $\mathrm{D}$, with individuals not taking vitamin supplements. Thus, there was an increase in the methylation within the promoter region of the SLC25A24 gene. SCLC25A24 encodes for the Calcium-binding mitochondrial carrier protein SCaMC-, one of five ATP-Mg/P ${ }_{\mathrm{i}}$ carriers with the vital function to exchange ATP-Mg or ADP for phosphate across the mitochondrial inner membrane. It has been shown that SLC25A24 mutations lead to impaired mitochondrial ATP synthesis and cause hyperpolarization and increased proton leakage, which results in decreasing energy metabolism [27]. In addition, genetic analyses in humans and mouse models have suggested that SLC25A24 acts as an essential regulator of body fat mass and adipogenesis [28]. In line with our results, SLC25A24 has been reported as a primary vitamin D-activated target.

Changes in the expression levels in the TSS of the gene were observed within four hours of stimulation with 1,25(OH)D in human monocytes [29]. The previous study and our results indicate a role of vitamin D in regulating transcriptional levels of SLC25A24. 
Only a few studies have examined the relationship between vitamin D supplementation and methylation patterns. Nair-Shalliker et al. showed that exposure to sunlight was inversely associated with global DNA methylation and that vitamin D levels did not influence this association [13]. Chavez Valencia et al. exposed primary human blood mononuclear cells to calcitriol for up to 120 hours and showed that DNA methylation patterns did not change in response to vitamin D treatment [12]. Tapp et al. investigated the effects of several nutritional factors and age-related DNA methylation in the human rectal mucosa and reported a weak positive correlation between vitamin $D$ and LINE-1 methylation [9].

We have also evaluated the influence of other lifestyle habits on methylation levels, such as general vitamins intake, eating habits (fish intake), and physical activity. In general, the global methylation patterns did not change according to the lifestyle factors evaluated in this study. However, two DMPs in intergenic regions were found when comparing individuals who exerted moderate-high physical activity with those who exerted very low or almost no physical activity. Several studies have shown that both lifelong (chronic) and acute physical activity affect DNA methylation in the skeletal muscle, blood, and saliva, and several epigenetic markers have been suggested [18, 30-32]. However, these studies reported epigenetic modifications as outcomes of exercise intervention. Our study is a cross-sectional study, where hours per week of physical activity and relative intensities of the exercise were self-reported in a questionnaire. Thus, we recognize that the study participants' estimations of the intensity of the physical activity might affect this type of retrospective study.

In a previous study, Rondanelli et al. could show that combining vitamin D intake and physical activity resulted in synergistic effects in counteracting sarcopenia in the elderly, such as increasing muscle and strength [21]. Moreover, physical activity has been associated with an increase of circulating 25(OH)D in the elderly $[22,23]$. The isolated effects of vitamin $D$ supplementation and physical activity on methylation have been evaluated previously, but not their combined effects. High levels of physical activity together with vitamin D supplementation was associated with DMPs in 221 genes and 108 intergenic regions. Out of these DMPs, $21 \%$ were located within promoter regions suggesting a role in transcriptional regulation. Functional analyses, of the genes corresponding to all the DMPs, showed that a great proportion of the genes were related to the regulation of the transcription by RNA polymerase II.

Interestingly, some genes containing the hypermethylated DMPs were involved in regulating the vitamin D receptor pathway. Thus, we speculate that high physical activity may increase vitamin $D$ bioavailability by increasing the binding of vitamin D with DBP and, in the end, regulating the transcription of genes related to the vitamin $\mathrm{D}$ receptor. Other genes containing the hypermethylated DMPs were involved in Wnt-signaling and pathways implicated in cancer development. The increase of methylation levels in these genes point to an inhibition of the transcriptional activity in cancer and Wnt-signaling pathways. These results are in line with previous studies, where $1,25(\mathrm{OH}) \mathrm{D}_{3}$ and physical activity inhibited activation of the Wnt/ $\beta$-catenin signalling, a pathway frequently hyperactive in cancer $[33,34]$.

We also analysed the methylation status of 2,113 CpG sites in 80 vitamin D-related genes, including wellknown receptors, metabolic enzymes, and primary activated targets of vitamin $D$, identified from the 
literature. Thus, we found an inverse correlation between $R X R A$ methylation and age. Intriguingly, a previous study using computational networks identified that one of the most critical key nodes related to chronological age across multiple tissue types was RXRA methylation [35].

The methylation levels of the vitamin D-related genes examined here did not change in individuals with a vitamin D-containing diet, vitamin D supplementation, or sun exposure. Results from previous studies of methylation of vitamin $D$ receptors and associated metabolic enzymes in response to vitamin $D$ supplementation are inconsistent and contradictory [14-16]. Zhou et al. reported that the methylation status of CYP2R1 and CYP24A1 were negatively associated with 25(OH)D baseline plasma levels [16]. However, these associations were weak and disappeared when the prediction was corrected for vitamin $D$ intake [16]. On the other hand, Beckett et al. showed that calcium intake, age, sex, BMI, cigarette smoking history, alcohol intake, and cumulative irradiance increased the association with 25(OH)D plasma levels [15]. However, this study was only conducted on peripheral blood cells, and the cohort was of limited size [15].

Several other factors could have influenced the results of our analyses, such as gender, age, smoking, and alcohol consumption. When analysing differences in methylation depending on gender, a total of 4,329 DMPs and 591 DMRs were identified. In the case of smoking, a few DMPs were found when the group of higher cigarette consumption was compared with the group that did not smoke. Several studies have demonstrated an association between age and alcohol consumption [36-39], but in this cohort, no associations were found when comparing age and alcohol consumption. This could be due to a narrow age range within the cohort, and that the group that reported high consumption of alcohol (SD/week) was reduced in size, respectively.

In summary, we have examined the influence of lifestyle and vitamin D intake on global DNA methylation patterns in an elderly cohort in Southwest of Sweden. An association between age and the methylation of RXRA was found, which confirms previous observations of epigenetic clocks. We could also show that the combination of vitamin $D$ supplementation and a high level of physical activity elicited changes in DNA methylation in genes regulating the vitamin D receptor pathway, implying that physical activity and vitamin $\mathrm{D}$ supplements may lead to transcription of genes related to the vitamin $\mathrm{D}$ receptor and pathways. However, since the group containing individuals taking vitamin D supplements and exerting high levels of physical activity was relatively small compared to the group of individuals not taking vitamin D supplements and exerting low levels of physical activity, these results need to be verified in a comprehensive interventional study. Also, since the samples for DNA extraction were collected from the saliva, local effects on DNA methylation in the skeletal muscle tissue cannot be detected. On the other hand, our results demonstrate global methylation effects from physical activity and vitamin $D$ supplementation.

\section{Conclusions}


In this study, we show that lifestyle factors, i.e., moderate to high levels of physical activity combined with the intake of vitamin D supplements, affect DNA methylation in genes regulating the vitamin-D-receptor pathway and pathways associated with cancer initiation and development. Vitamin D deficiency is abundant among the elderly, and considering different choices in lifestyle may substantially contribute to healthy aging. To our knowledge, this is the first study showing epigenetic changes associated with the combined effects of vitamin D supplementation and high physical activity in an elderly population.

\section{Methods}

\section{Study Participants}

The elderly cohort analysed in this study included community-dwelling individuals recruited in collaboration with the association Active Seniors (Aktiva seniorer) - a nationwide independent political association in Sweden for older adults. A total of 800 individuals in Southwest Sweden, at age 70 to 95, were invited to participate in the study, as described in [26]. At the seminars, the participants were informed about the study and asked to participate. Altogether, 530 participants answered a questionnaire including background data and different lifestyle habits (Additional file 1). Out of the 530 participants, 10 individuals choose to participate solely in the questionnaire study. Thus, saliva samples for subsequent DNA extraction were collected from 520 individuals and kept in a freezer at $-20^{\circ} \mathrm{C}$. Of the 520 saliva samples, 277 samples were randomly selected for methylation analyses. After data processing three samples were removed and 274 samples remained. The background data of the cohort participants in the study was pre-processed (three samples were removed) and are presented in Table 3. 
Table 3

Background data among participants in the methylation study $(\mathrm{n}=274)$.

\section{General characteristics}

\begin{tabular}{|c|c|c|c|}
\hline Gender & Female $(n=188)$ & Male $(n=81)$ & Total $(n=269, n / a=5)$ \\
\hline Age (mean $\pm S D)$ & $75.1 \pm 5.8$ & $76.1 \pm 4.9$ & $75.4 \pm 5.5$ \\
\hline \multicolumn{4}{|l|}{ Medical data } \\
\hline \multicolumn{4}{|c|}{ Self-reported health status (SRHS) } \\
\hline Very good & 84 & 31 & 115 \\
\hline Satisfactory & 94 & 47 & 141 \\
\hline Unsatisfactory & 4 & 4 & 8 \\
\hline \multicolumn{4}{|l|}{ Medicine - Lipids } \\
\hline Yes & 130 & 52 & 182 \\
\hline No & 35 & 20 & 55 \\
\hline \multicolumn{4}{|c|}{ Medicine - Blood pressure } \\
\hline Yes & 108 & 39 & 147 \\
\hline No & 70 & 40 & 110 \\
\hline \multicolumn{4}{|l|}{ Medicine - Heart } \\
\hline Yes & 133 & 51 & 184 \\
\hline No & 29 & 22 & 51 \\
\hline
\end{tabular}

\section{Study Groups}

The cohort was stratified into study groups based on participants' answers to general characteristics (age and gender), medical data (Table 3 ) and questions about different lifestyle factors, including vitamin supplementations, smoking and alcohol habits, physical activity and sunbathing habits. (Table S1, Additional file 2). Vitamin D intake was evaluated from the vitamin $D$ supplementation and dietary vitamin D (Fish and seafood frequency, Table S1, Additional file 2). To estimate potential vitamin D synthesis in the skin, sunlight exposure and use of sunscreen were analysed (Sun exposure and use of sunscreen, Table S1B, Additional file 2). Physical activity during the whole year was evaluated from the combination of answers to physical activity in summer and in winter (Physical activity during summer and winter, Table S1A) 


\section{DNA methylation analysis}

DNA was extracted from $1 \mathrm{~mL}$ of saliva using SalivaGene Collector and PSP SalivaGene DNA Kit (Stratec, Berlin, Germany). DNA quality, purity, and concentration were assessed spectrophotometrically using Nanodrop. The extracted DNA was submitted for genome-wide array analysis to Swegene Centre for Integrative Biology at Lund University (SCIBLU), Sweden. Global methylation patterns were measured using the Illumina Infinium DNA Methylation EPIC 850k BeadChip array to interrogate methylation in 850,000 probes or 285,000 genes/markers, respectively, across the genome.

The raw intensity files (idat) were exported from Genome Studio and imported into R (R Core Team 2021) for further analyses. Annotation, pre-processing, quality control (QC), and differential methylation analyses were performed with Miodin (MultI-Omics Data INtegration) package [40]. Pre-processing consisted of filtering probes (non-CG, close to SNPs, cross-hybridizing, and low-variance) and a BetaMixture Quantile (BMIQ) normalization and cell composition correction. After pre-processing, 198,550 probes were filtered out and 667,259 remained. None of the samples were removed during pre-processing and QC. Potential variation or batch effects on the methylation data were assessed by principal component analysis (PCA). Three samples were considered outliers and removed after PCA, and 274 remained for the following analysis.

\section{Differential methylation analysis}

Differential methylation analysis was performed for all the study groups associated with self-reported medical data (Table 3) and lifestyle habits (Table S1, Additional file 2). Moreover, these analyses were done for the combination of different lifestyle factors with vitamin D (dietary, supplementation, and synthesis). The differential methylation analyses were conducted in Miodin with the DMRcate package [41]. Gender, age, smoking and alcohol (SD and frequency), were included as covariates in the analyses. $P$ values were adjusted for multiple testing with Benjamini-Hochberg correction. Methylated probes or CpG sites, and regions, with a difference in beta value $(\beta)$ between groups $\geq 0.2$ or $\leq-0.2$ and false discovery rate $(F D R)<0.05$, were considered differentially methylated. DMPs were considered "hypomethylated" if $\beta$ $\leq-0.2$, and "hypermethylated" if $\beta \geq 0.2$. Genes overlapping with the DMPs were used as input for the functional annotation and network analyses.

\section{Functional annotation and associated network analysis}

Functional annotation analysis was performed using the GO enrichment analysis with PANTHER 16.0 [42, 43]. The main regulated pathways were extracted from the pathway databases KEGG (Kyoto Encyclopedia of Genes and Genomes) and WikiPathways in GeneCodis 4.0 [44]. Genes overlapping with differentially hypomethylated and hypermethylated probes were input into the different tools separately and together. Network analysis was performed using GeneMANIA prediction server (University of Toronto, www.genemania.org) [45]. GeneMANIA was used to detect related genes, potential interactions, and associated networks to the input genes from datasets with available genomics and proteomics data. 
Maximal resultant genes were set to 30 and maximal resultant attributes to 20 . Functionally enriched pathways and results from the associated network analysis were considered significant if FDR < 0.05 .

\section{Analysis of methylation status of vitamin D-related genes}

To examine the association between methylation levels in vitamin-D-related genes with each study group, a total of 80 vitamin D-related genes corresponding to 2,113 CpG sites were selected for further analyses. The selected genes included the vitamin D receptors (VDR and PDIA3), metabolic enzymes (CYP27A1, CYP27B1, CYP2R1, and CYP24A1), secondary activated receptors (RXRA and TRPV6), vitamin D binding protein $(D B P)$, and primary activated targets of vitamin $\mathrm{D}$, previously reported in literature and databases (Table S2, Additional file 2). Analysis of the association of methylation levels of vitamin D genes with non-binary factors was performed using Spearman's correlation test. Differences in methylation levels for binary factors were assessed with Welch's two samples t-test considering unequal variances. Methylation values were corrected for batch effects of gender. $P$ values were adjusted for multiple testing with Benjamini-Hochberg correction.

\section{Abbreviations}

CYP2R1

Calciol-25-hydroxylase

CYP27B1

Calcidiol-1a-hydroxylase

CYP24A1

24-hydroxylase

DBP

Vitamin D binding protein

DMP

Differentially methylated probe

DMR

Differentially methylated region

FDR

False discovery rate

PCA

Principal component analysis

PDIA3

Protein disulfide-isomerase A3

SHRS

Self-reported health status

TGF- $\beta$

Transforming growth factor beta

TSS 
Transcription start site

VDR

Vitamin D receptor

\section{Declarations}

\section{Ethics approval and consent to participate}

This study followed the Declaration of Helsinki [46] and was approved by the Regional Ethical Review Board in Gothenburg (Dnr: 989-13). Written informed consent was obtained from all participants.

\section{Consent for publication}

Not applicable

\section{Availability of data and materials}

The DNA methylation data have been deposited in ArrayExpress database at EMBL-EBI [www.ebi.ac.uk/arrayexpress] under accession number E-MTAB-11445.

\section{Competing interests}

The authors declare that they have no competing interests.

\section{Funding}

The University of Borås and the University of Skövde provided with funding for the different experiments performed in this study. Jönköping University supplied with the necessary resources to carry out this investigation.

\section{Authors'contributions}

MADC conducted the experimental design of this specific study, data acquisition, statistical analyses, interpretation of the results and preparation of the manuscript. BU assisted with the experimental design and data analysis, revised and approved the manuscript final version. PB and MF has contributed to revision and final approval of the manuscript. FS and SK conceived the study, experimental design and contributed to interpretation of the results, preparation, revision, and approval of the manuscript. All authors read and approved the final manuscript.

\section{Acknowledgements}

We thank the "Aktiva seniorer" association in Sweden for their collaboration in this study. The computations in this study were enabled by resources provided by the Swedish National Infrastructure for Computing (SNIC) at Uppsala Multidisciplinary Center for Advanced Computational Science (UPPMAX), partially funded by the Swedish Research Council through grant agreement no. 2018-05973. 


\section{References}

1. Nair, R. and A. Maseeh, Vitamin D: The "sunshine" vitamin. J Pharmacol Pharmacother, 2012. 3(2): p. 118-26.

2. Alegría-Torres, J.A., A. Baccarelli, and V. Bollati, Epigenetics and lifestyle. Epigenomics, 2011. 3(3): p. 267-77.

3. Calcium, I.o.M.U.C.t.R.D.R.I.f.V.D.a., Dietary Reference Intakes for Calcium and Vitamin D, ed. C.L.T. A Catharine Ross, Ann L Yaktine, and Heather B Del Valle. 2011: Washington (DC): National Academies Press (US).

4. Holick, M.F., Vitamin D: a d-lightful solution for health. J Investig Med, 2011. 59(6): p. 872-80.

5. Hossein-nezhad, A. and M.F. Holick, Vitamin D for health: a global perspective. Mayo Clin Proc, 2013. 88(7): p. 720-55.

6. Meehan, M. and S. Penckofer, The Role of Vitamin D in the Aging Adult. J Aging Gerontol, 2014. 2(2): p. $60-71$.

7. Cherniack, E.P., et al., Hypovitaminosis D in the elderly: from bone to brain. J Nutr Health Aging, 2008. 12(6): p. 366-73.

8. Prosser, D.E. and G. Jones, Enzymes involved in the activation and inactivation of vitamin D. Trends Biochem Sci, 2004. 29(12): p. 664-73.

9. Tapp, H.S., et al., Nutritional factors and gender influence age-related DNA methylation in the human rectal mucosa. Aging Cell, 2013. 12(1): p. 148-55.

10. Seuter, S., et al., Dynamics of 1a,25-dihydroxyvitamin D3-dependent chromatin accessibility of early vitamin D receptor target genes. Biochim Biophys Acta, 2013. 1829(12): p. 1266-75.

11. Chen, L., et al., Effects of Vitamin D3 Supplementation on Epigenetic Aging in Overweight and Obese African Americans With Suboptimal Vitamin D Status: A Randomized Clinical Trial. J Gerontol A Biol Sci Med Sci, 2019. 74(1): p. 91-98.

12. Chavez Valencia, R.A., et al., In vitro exposure of human blood mononuclear cells to active vitamin $D$ does not induce substantial change to DNA methylation on a genome-scale. J Steroid Biochem Mol Biol, 2014. 141: p. 144-9.

13. Nair-Shalliker, V., et al., The association between personal sun exposure, serum vitamin $D$ and global methylation in human lymphocytes in a population of healthy adults in South Australia. Mutat Res, 2014. 765: p. 6-10.

14. Fetahu, I.S., J. Höbaus, and E. Kállay, Vitamin D and the epigenome. Frontiers in Physiology, 2014. 5(164).

15. Beckett, E.L., et al., Relationship between methylation status of vitamin D-related genes, vitamin D levels, and methyl-donor biochemistry. Journal of Nutrition \& Intermediary Metabolism, 2016. 6: p. 8-15.

16. Zhou, Y., et al., DNA methylation levels of CYP2R1 and CYP24A1 predict vitamin D response variation. J Steroid Biochem Mol Biol, 2014. 144 Pt A: p. 207-14. 
17. Langhammer, B., A. Bergland, and E. Rydwik, The Importance of Physical Activity Exercise among Older People. Biomed Res Int, 2018. 2018: p. 7856823.

18. Recchioni, R., et al., Epigenetic effects of physical activity in elderly patients with cardiovascular disease. Exp Gerontol, 2017. 100: p. 17-27.

19. Sellami, M., et al., Regular, Intense Exercise Training as a Healthy Aging Lifestyle Strategy: Preventing DNA Damage, Telomere Shortening and Adverse DNA Methylation Changes Over a Lifetime. Front Genet, 2021. 12: p. 652497.

20. Barrès, R., et al., Acute exercise remodels promoter methylation in human skeletal muscle. Cell Metab, 2012. 15(3): p. 405-11.

21. Rondanelli, M., et al., Whey protein, amino acids, and vitamin D supplementation with physical activity increases fat-free mass and strength, functionality, and quality of life and decreases inflammation in sarcopenic elderly. Am J Clin Nutr, 2016. 103(3): p. 830-40.

22. Hall, J.T., et al., The relationship between physical activity and vitamin D status in postpartum lactating and formula-feeding women. J Steroid Biochem Mol Biol, 2018. 177: p. 261-265.

23. Scott, D., et al., A prospective study of the associations between 25-hydroxy-vitamin D, sarcopenia progression and physical activity in older adults. Clin Endocrinol (Oxf), 2010. 73(5): p. 581-7.

24. Mason, R.S., et al., The Role of Skeletal Muscle in Maintaining Vitamin D Status in Winter. Curr Dev Nutr, 2019. 3(10): p. nzz087.

25. Bollati, V., et al., Decline in genomic DNA methylation through aging in a cohort of elderly subjects. Mech Ageing Dev, 2009. 130(4): p. 234-9.

26. Gillsjo, C., et al., Lifestyle's influence on community-dwelling older adults' health: A mixed-methods study design. Contemp Clin Trials Commun, 2021. 21: p. 100687.

27. del Arco, A. and J. Satrustegui, Identification of a novel human subfamily of mitochondrial carriers with calcium-binding domains. J Biol Chem, 2004. 279(23): p. 24701-13.

28. Urano, T., et al., SLC25A24 as a novel susceptibility gene for low fat mass in humans and mice. J Clin Endocrinol Metab, 2015. 100(4): p. E655-63.

29. Nurminen, V., S. Seuter, and C. Carlberg, Primary Vitamin D Target Genes of Human Monocytes. Frontiers in Physiology, 2019. 10(194).

30. Bryan, A.D., et al., Physical activity and differential methylation of breast cancer genes assayed from saliva: a preliminary investigation. Ann Behav Med, 2013. 45(1): p. 89-98.

31. Sailani, M.R., et al., Lifelong physical activity is associated with promoter hypomethylation of genes involved in metabolism, myogenesis, contractile properties and oxidative stress resistance in aged human skeletal muscle. Sci Rep, 2019. 9(1): p. 3272.

32. Robson-Ansley, P.J., et al., Dynamic changes in dna methylation status in peripheral blood Mononuclear cells following an acute bout of exercise: Potential impact of exercise-induced elevations in interleukin-6 concentration. J Biol Regul Homeost Agents, 2014. 28(3): p. 407-17. 
33. Malcomson, F.C., Mechanisms underlying the effects of nutrition, adiposity and physical activity on colorectal cancer risk. Nutrition Bulletin, 2018. 43(4): p. 400-415.

34. Leal, M.L., et al., Effect of different resistance-training regimens on the WNT-signaling pathway. European Journal of Applied Physiology, 2011. 111(10): p. 2535-2545.

35. Palla, G., et al., Hierarchy and control of ageing-related methylation networks. PLoS Comput Biol, 2021. 17(9): p. e1009327.

36. Zaghlool, S.B., et al., Association of DNA methylation with age, gender, and smoking in an Arab population. Clin Epigenetics, 2015. 7(1): p. 6.

37. Jiang, S. and Y. Guo, Epigenetic Clock: DNA Methylation in Aging. Stem Cells Int, 2020. 2020: p. 1047896.

38. Liu, C., et al., A DNA methylation biomarker of alcohol consumption. Mol Psychiatry, 2018. 23(2): p. 422-433.

39. Jones, M.J., S.J. Goodman, and M.S. Kobor, DNA methylation and healthy human aging. Aging Cell, 2015. 14(6): p. 924-32.

40. Ulfenborg, B., Vertical and horizontal integration of multi-omics data with miodin. BMC Bioinformatics, 2019. 20(1): p. 649.

41. Peters, T.J., et al., De novo identification of differentially methylated regions in the human genome. Epigenetics \& Chromatin, 2015. 8(1): p. 6.

42. Mi, $\mathrm{H}$., et al., PANTHER version 16: a revised family classification, tree-based classification tool, enhancer regions and extensive API. Nucleic Acids Research, 2020. 49(D1): p. D394-D403.

43. Ashburner, M., et al., Gene ontology: tool for the unification of biology. The Gene Ontology Consortium. Nat Genet, 2000. 25(1): p. 25-9.

44. Tabas-Madrid, D., R. Nogales-Cadenas, and A. Pascual-Montano, GeneCodis3: a non-redundant and modular enrichment analysis tool for functional genomics. Nucleic Acids Res, 2012. 40(Web Server issue): p. W478-83.

45. Zuberi, K., et al., GeneMANIA prediction server 2013 update. Nucleic Acids Res, 2013. 41(Web Server issue): p. W115-22.

46. Mata-Granados, J.M., et al., Vitamin D insufficiency together with high serum levels of vitamin $A$ increases the risk for osteoporosis in postmenopausal women. Arch Osteoporos, 2013. 8: p. 124.

\section{Figures}


A
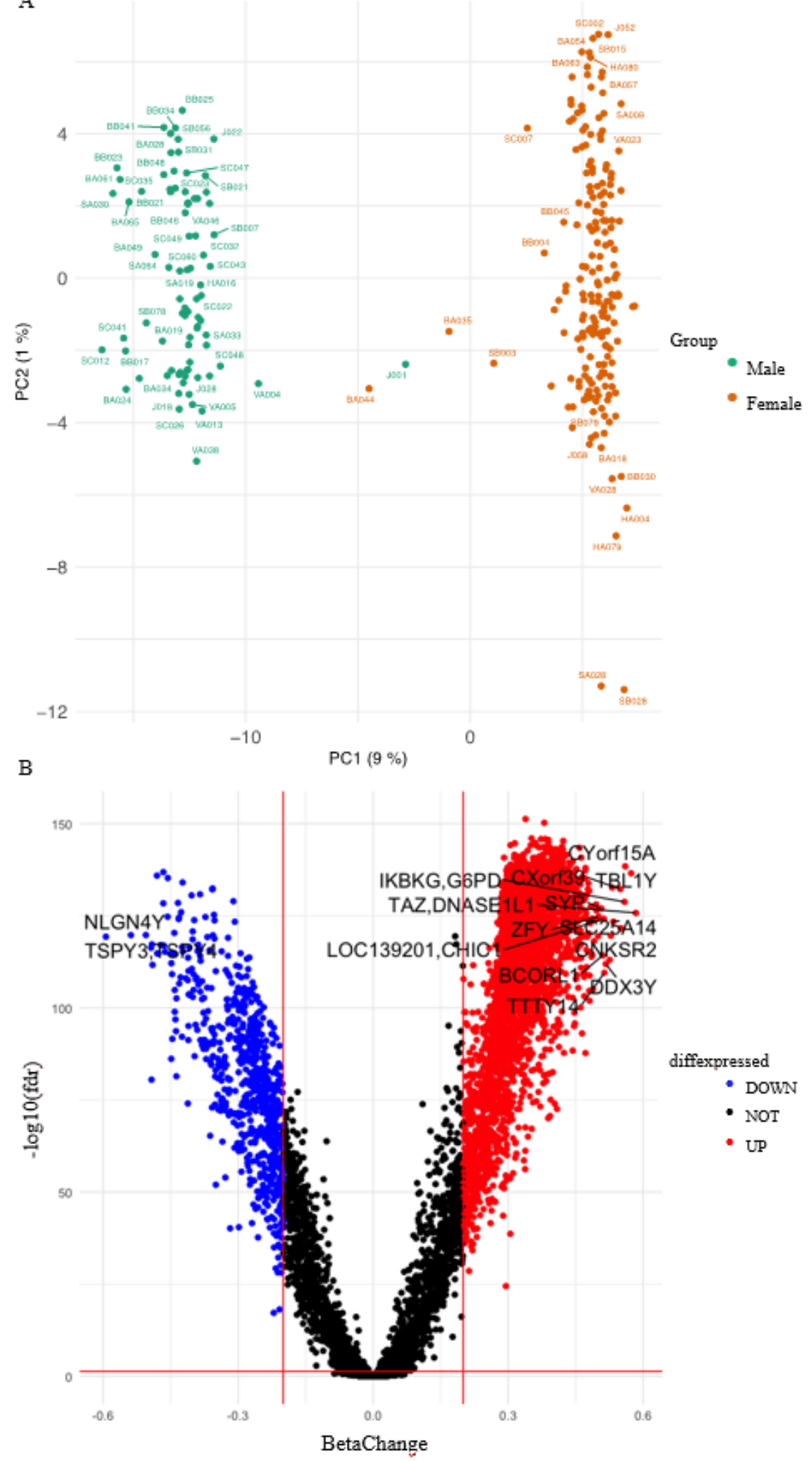

Figure 1

A. PCA on pre-processed methylation data showing differences between female and male individuals in the study $(n=269, n / a=5)$. B: Volcano Plot representing DMPs between females $(n=188)$ and males $(n=81)$. Red: Hypermethylated probes: $b>0.2$ and FDR<0.05. Blue: Hypomethylated probes: $b<-0.2$ and FDR<0.05. Black: Non-significant results. Gene names are shown for DMPs with $b>0.5$ and FDR $<0.0001$, and DMPs with $b<-0.5$ and FDR $<0.0001$. 
A

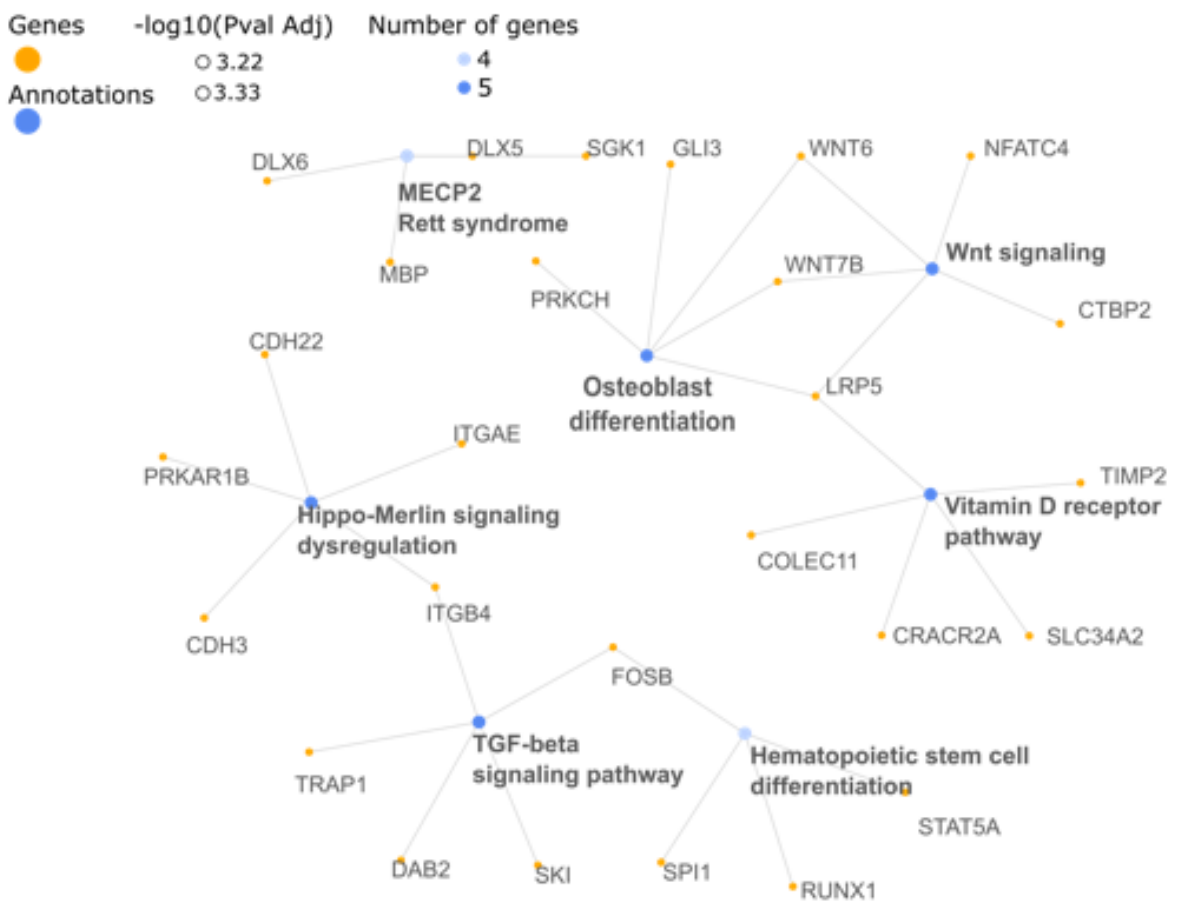

B

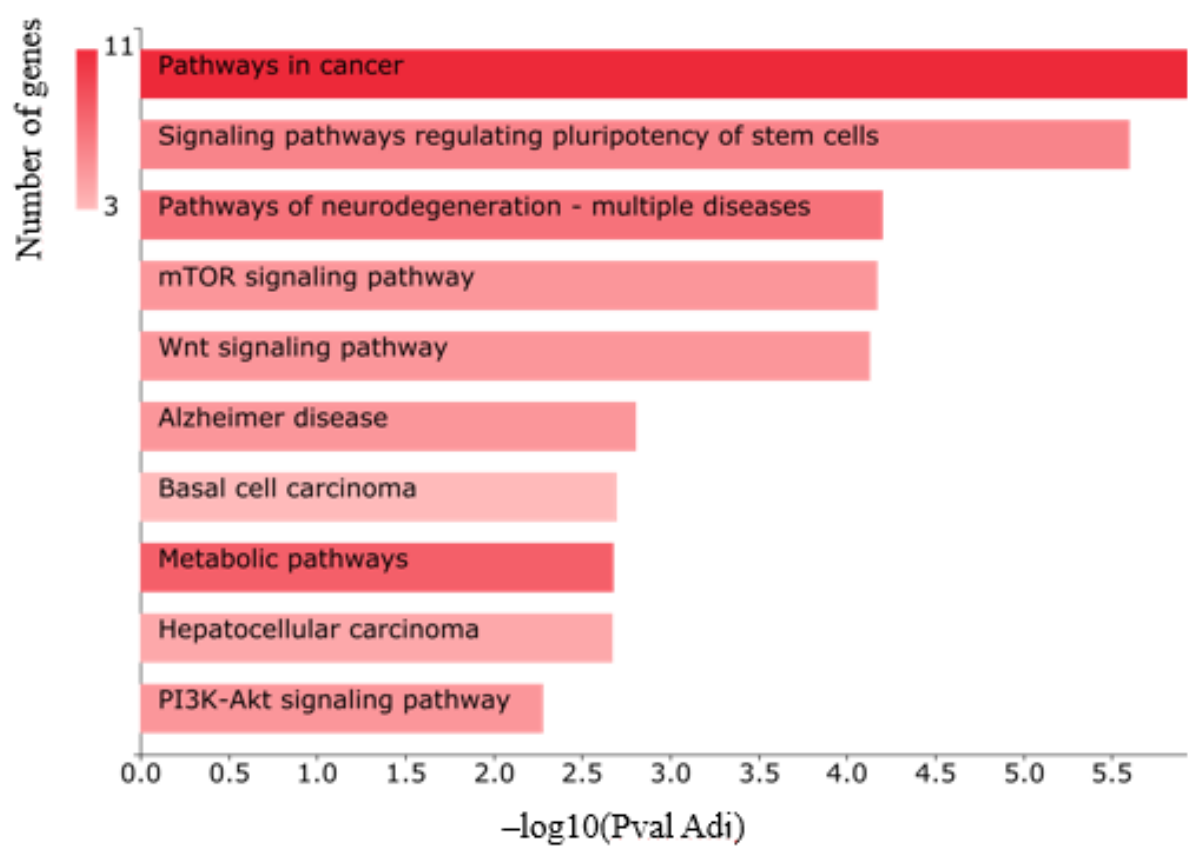

\section{Figure 2}

Main regulated pathways related to genes with differentially hypermethylated probes found in the vitamin $D$ and high physical activity group. A. Top 7 significant regulated pathways obtained with Wikipathways 
in GeneCodis, adjusted $\mathrm{P}$ value $<0.05$. B. Top 10 significant regulated pathways obtained with KEGG, adjusted $P$ value $<0.05$.
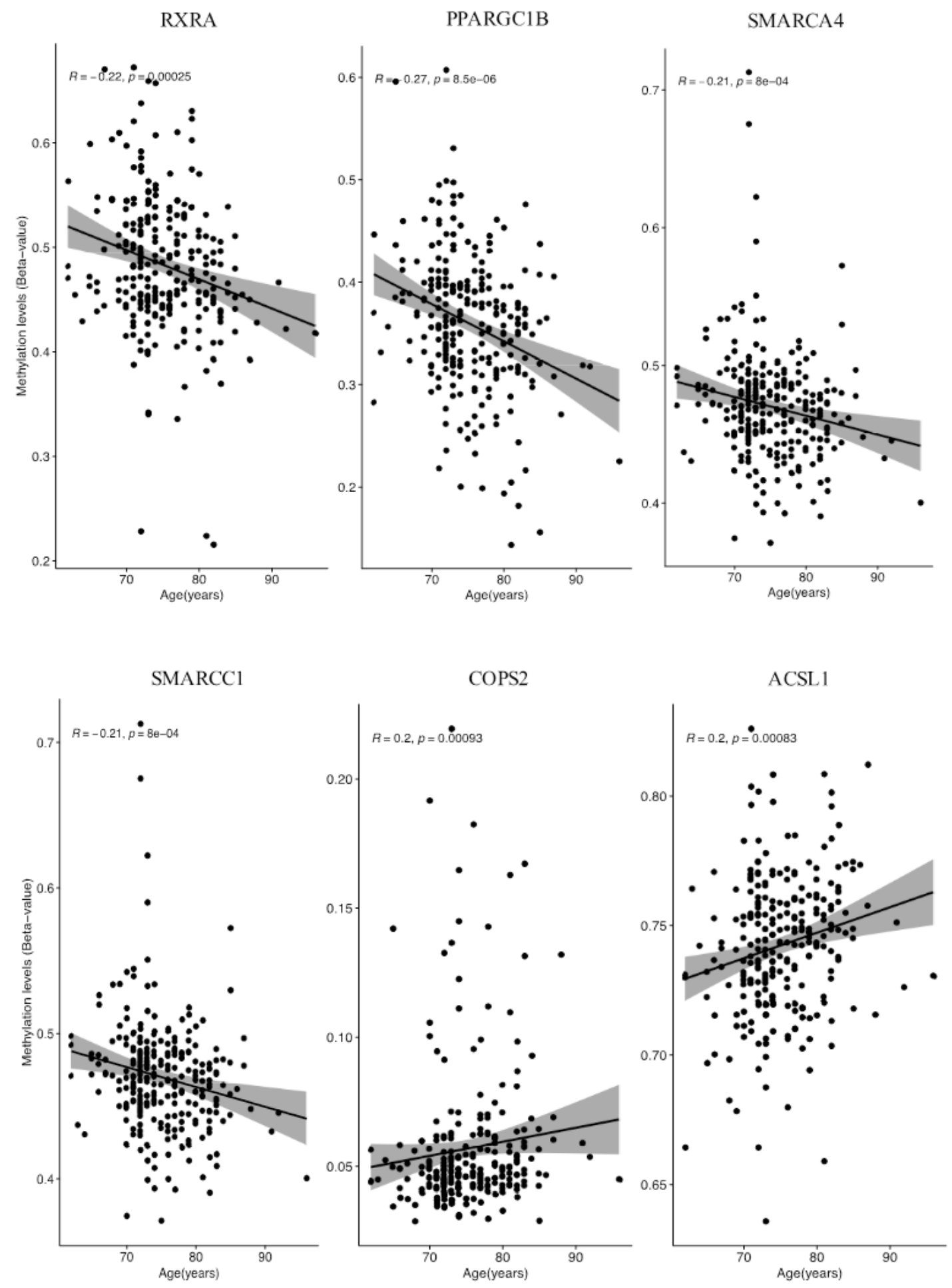

Figure 3 
Association of methylation levels of CpGs at RXRA, PPARGC1B, SMARCA4, SMARCC1, COSP2, and ACSL 1; and age of the individuals in the study. Association was evaluated by Spearman's correlation method (r2, $P$ value $<0.05)$.

\section{Supplementary Files}

This is a list of supplementary files associated with this preprint. Click to download.

- Additionalfile1.docx

- Additionalfile2.docx

- Additionalfile3.docx

- Additionalfile4.docx 